\title{
Increased Mpv and Pdw in Early Detection of Diabetic Neuropathy, Even if it is the Cause or Result of Microvascular Complication
}

\section{Sonmezler A* \\ Department of Neurology, Adana City Research and Training Hospital, Turkey}

*Corresponding author: Abdurrahman Sonmezler, Department of Neurology, Adana City Research and Training Hospital, Adana-Turkey, Email: sonmezlera@gmail.com

\section{Mini Review \\ Volume 5 Issue 1}

Received Date: January 16, 2020

Published Date: January 24, 2020

DOI: $10.23880 /$ accmj-16000168

\section{Abstract}

Diabetic neuropathy is a microvascular complication that is one of the causes of mortality and morbidity. An increasing number of studies point to the association of mean platelet volume (MPV) and platelet distribution width (PDW) with microvascular complications in diabetic neuropathy patients. MPV, PDW can therefore be used as simple and cost-effective predictive parameters of platelet activation to monitor and predict the risk of vascular complications. Early diagnosis of neuropathy will ensure significant success in preventing complications.

Keywords: Diabetic neuropathy; Mean platelet volume; Platelet distribution width

Abbreviations: DM: Diabetes Mellitus; MPV: Mean Platelet Volume; PDW: Platelet Distribution Width

\section{Introdution}

Diabetes Mellitus (DM) is a metabolic disease which is a major global health problem due to its high prevalence and morbidity.Diabetes Mellitus is a metabolic syndrome presenting with hyperglycemia causing macrovascular and microvascular complications. Platelet hyperactivity has been associated with a high risk of vascular disease. Increased mean platelet volume (MPV) and platelet distribution width (PDW) were found to be due to the cause of thromboembolic complications. Diabetic neuropathy is a microvascular complication that is one of the causes of mortality and morbidity .MPV is an indicator of the average size and activity of platelets. Larger platelets are more enzymatically and metabolically active and have higher thrombotic ability than small platelets indicated by increased MPV. PDW reflects how uniform the size of platelets is. Activated platelets with increased number and size of pseudopodia differ in size, in which case PDW varies. The prothrombotic stage of the platelet can easily be detected early using hematological analysers through these platelet parameters $[1,2]$.

Although the study designs, number of patients, age groups, and clinical characteristics are different, an increasing number of studies point to the association of MPV and PDW with microvascular complications in diabetic neuropathy patients. In a study evaluating 125 patients, MPV and PDW values were higher in patients with diabetes and complications. Platelet indices were also compared to the severity of neuropathy using the NDS (Neuropathy Disability Score), and it was found that MPV, PDW had a positive correlation with the NDS(2). In a study evaluating 280 patients, MPV,PDW and PLCR were significantly higher in diabetics compared to non diabetics. Among the diabetics, MPV,PDW and PLCR were higher in those with complications (macro or microvascular) as compared to those without complications [3]. In a study evaluating 300 patients, MPV and PDW were reported to be predictive biomarkers of diabetic vascular complications. It was emphasized that this condition was more significant in microvascular complications than in macrovascular complications [4]. 
Mean platelet volume (MPV), platelet distribution width (PDW) are considered important markers of platelet activation, which can be easily measured as part of a whole blood count. MPV, PDW can therefore be used as simple and cost-effective predictive parameters of platelet activation to monitor and predict the risk of vascular complications. However, increased MPV, PDW albeit as a cause or result of vascular complications, should be investigated further. Since neuropathy developed in diabetic patients is an important cause of morbidity and mortality, early diagnosis of neuropathy will ensure significant success in preventing complications.

\section{References}

1. Sushma KL, Rangaswamy M (2017) Study of Platelet
Indices in Type 2 Diabetic Patients and Its Correlation with Vascular Complications. Annals of Pathology and Laboratory Medicine 4(5).

2. Walinjkar RS, Khadse S, Kumar S, Bawankule S, Acharya $S$ (2019) Platelet Indices as a Predictor of Microvascular Complications in Type 2 Diabetes. Indian J Endocrinol Metab 23(2): 206-210.

3. Kumari S, Potekar RM (2017) A Study of Platelet Indices inType 2 Diabetes Mellitus Patients. Indian Journal of Hematology and Blood Transfusion 34(1): 1-6.

4. Buch A, Kaur S, Nair R, Jain A (2017) Platelet volume indices as predictive biomarkers for diabetic complications in Type 2 diabetic patients.J Lab Physicians 9(2): 84-88. 